\title{
Color solitons in the extended chiral group $\mathrm{E} \chi$
}

Victor V. Novozhilov ${ }^{1} 2$

St.Petersburg State University

E-mail: vnovozhilovemail.ru

We consider extended chiral group $E \chi$ and investigate whether soliton-skyrmion with diquark parameters can be formed within sympletic subgroup of $E \chi$. We find that classically stable, finite mass topological solitons exist in the extended chiral group $E \chi$. In the case of three colors, two flavors their status is described by the chiral group $O(3)_{\tilde{L}} \times O(3)_{\tilde{R}}$. The vacuum background gluon field defines also an asymptotic behavior of the shape function $F(R)$; the field should be chromomagnetic. 


\section{Contents}

\section{Introduction}

The extended chiral group $E \chi[1,2]$ was introduced in the bosonisation approach $[3,4,5]$ to derive the effective action of diquark fields. The corresponding extended chiral transformation depends both on pseudoscalar and diquark fields as parameters. While at the classical level the chiral symmetry is broken by quark mass, the extended chiral $(E \chi)$ symmetry is broken by quark mass and gluon fields. $E \chi$-group is $U(2 N)$ for $N$ internal degrees of freedom, $N=N_{c} N_{f}$. Non-anomalous (measure preserving) generators span the Lie algebra of $O(2 N)$, anomalous generators belong to the coset $U(2 N) / O(2 N)$. Anomalous generators describe chiral rotations and transformations with diquark variables ("diquark" rotations), non-anomalous part consists out of gauge transformations and combined chiral "diquark" rotations. It was assumed that $E \chi$-symmetry breaking due to quark masses and gluon fields is soft in the sense that the action for bosonised diquark fields can be obtained by integrating corresponding $E \chi$-anomaly. Colorless chiral fields after bosonisation give rise to Goldstone particles - pseudoscalar mesons. At low energies bosonised diquark parameters of $E \chi$-transformations with quantum numbers of lightest $J^{P}=0^{+} u d$-diquark was treated as a Goldstone-like particle. The $E \chi$-group in this case is $S U(4)$, non-anomalous transformations are just gauge transformations $S U(3) U(1)$ and the diquark Goldstone degrees of freedom belongs to $C P^{3}=S U(4) / S U(3) U(1)$. In the limit of vanishing gluon condensate and current quark masses the $u d$-diquark introduced a la Goldstone becomes massless.

Understanding world of color solitons is important in an non-perturbative approach to the low energy hadron physics. One of the problems is to include chiral anomalies $[6,7]$ in the total (gauge plus chiral, or anomalous) color space and to study changes of gauge space. In the gauge sector of $S U(2)$ QCD theory, color solitons were reported by Faddeev-Niemi $[8,9,10]$ and Cho et al. [11, 12, 13]. In the quark chiral sector, the Skyrmion model for constituent quarks (qualitons) was discussed by Kaplan [14, 15, 16], and it was shown $[17,18]$ that isolated color solitons (i.e. solitons on the background of vacuum gluon field) can be classically stabilized by the chromomagnetic vacuum field in the cases of two colors, one flavor and three colors, one flavor, their mass can be evaluated and intersoliton potential displays confinement behavior. A possibility of existence of a colour chiral solitons with 
baryon number $B=N_{F} / N_{C}$ was mentioned in the paper on colour bosonization [3], where baryon number current $B_{\mu}$ was derived

$$
B_{\mu}=\frac{N_{F}}{24 \pi^{2} N_{C}} \varepsilon_{\mu \nu \lambda \sigma} t r\left[U D^{\nu} U^{-1} U D^{\lambda} U^{-1} U D^{\sigma} U^{-1}-3 G^{\nu \lambda}\left(U D^{\sigma} U^{-1}-U^{-1} D^{\sigma} U\right)\right]
$$

The aim of this talk is to discuss quark and diquark type topological color solitons, existing in the extended chiral group $E \chi$. We consider isolated solitons which are defined as solitons in vacuum background field. We describe vacuum in a phenomenological way [20] through condensates and assume that cubic gluon condensate is zero.

\section{Colour chiral bosonization and effective action}

Chiral bosonization $[3,4]$ is a prescription for introducing chiral field, and integration of chiral anomaly is usually invoked, as a way to derive the chiral effective action.

Let us consider the Dirac lagrangian $L_{\psi}(G, A)$ with background gluon field $G_{\mu}$ and external colour field $A_{\mu}$ in the group $\mathrm{SU}(\mathrm{N}) \times S U(N)$

$$
L_{\psi}=i \bar{\psi}\left(\widehat{\partial}+\widehat{G}+\gamma_{5} \widehat{A}\right) \psi=\bar{\psi} D(G, A) \psi
$$

A chiral field $U$ is defined by the following transformation of Dirac fermions $\psi^{U}=\frac{1}{2}\left[\left(1-\gamma_{5}\right) U+\right.$ $\left(1+\gamma_{5}\right) \psi, U^{+} U=1$. The quark Lagrangian $L_{\psi}(G, A)$ remains invariant, if fields $G_{\mu}, A_{\mu}$ are transformed appropriately $L_{\psi}(G, A)=\overline{\psi^{U}} D\left(G^{U}, A^{U}\right) \psi^{U}$, where $U$-transformed fields are given by

$$
G_{\mu}^{U}+A_{\mu}^{U}=U\left(G_{\mu}+A_{\mu}\right) U^{-1}+U \partial_{\mu} U^{-1}, G_{\mu}^{U}-A_{\mu}^{U}=G_{\mu}-A_{\mu}
$$

Repeated transformation with the chiral field $U_{1}$ gives $\left(G_{\mu}^{U}\right)^{U_{1}}=G_{\mu}^{U_{1} U}$. These fields are not symmetrical / antisymmetrical with respect to left-right exchange. However, they are gauge transforms of vector and axial vector fields $\widetilde{G_{\mu}}, \widetilde{A}_{\mu}$ with the gauge function $\chi$ which is square root of $U$

$$
G_{\mu}^{U}=\chi \widetilde{G}_{\mu} \chi^{-1}+\chi \partial_{\mu} \chi^{-1}, A_{\mu}^{U}=\chi \widetilde{A}_{\mu} \chi^{-1}, \chi^{2}=U
$$

Under a gauge transformation $g$ the function $\chi$ transforms as $\chi^{\prime}=g \chi g^{-1}$.

The infinitesimal chiral transformation $g_{5}=1+\gamma_{5} \lambda$ acts in the following way

$$
\delta G_{\mu}=\left[A_{\mu}, \lambda\right], \delta A_{\mu}=D_{\mu} \lambda, \delta \psi=\gamma_{5} \lambda \psi, \delta \bar{\psi}=\bar{\psi} \gamma_{5} \lambda, \delta U=-(U \lambda+\lambda U)
$$

An important property of $U$-transformed fields $G^{U}, A^{U}, \psi^{U}, \overline{\psi^{U}}$ is that for them the chiral transformation $g_{5}$ is a non-chiral gauge transformation

$$
\delta G_{\mu}^{U}=-D_{\mu}\left(G^{U}\right) \lambda, \delta A_{\mu}^{U}=\left[\lambda, A_{\mu}^{U}\right], \delta \psi^{U}=\lambda \psi^{U}, \delta \bar{\psi}^{U}=-\bar{\psi}^{U} \lambda
$$

It follows that the Yang-Mills Lagrangian $L_{Y M}\left(G^{U}\right)=\frac{1}{2 g^{2}} \operatorname{tr} G_{\mu \nu}^{U}\left(G^{U}\right)^{\mu v}$ is invariant under chiral transformations . 
In order to find an effective action for the colour chiral field $U$ we study functional integrals

$$
\begin{gathered}
Z_{\psi}(G, A, R S)=\int D \bar{\psi} D \psi \exp i \int d x L_{\psi}(G, A)=\exp i W(G, A, R S) \\
Z_{\psi}\left(G^{U}, A^{U}, R S\right)=\int D \bar{\psi} D \psi \exp i \int d x L_{\psi}\left(G^{U}, A^{U}\right)=\exp i W\left(G^{U}, A^{U}, R S\right)
\end{gathered}
$$

which are also specified by a Regularization Scheme $R S$. They play the role of quantities $Z$ and $Z^{U}$ in definition of an effective action $W_{\text {eff }}(U)$. Thus, we obtain

$$
W_{e f f}(G, U, R S)=-i \ln \frac{Z_{\psi}(G, A, R S)}{Z_{\psi}\left(G^{U}, A^{U}, R S\right)}
$$

The usual way to calculate effective chiral action is to find an infinitesimal change $\delta_{U} W_{\text {eff }}$ (i.e.the anomaly) and integrate it up to $U$. We put $U=\exp \Theta$ and introduce the anomaly $A(x, \Theta)$

$$
A(x, \Theta)=\frac{1}{i} \frac{\delta \ln Z_{\psi}(\exp \Theta)}{\delta \Theta}
$$

Then

$$
W_{e f f}^{\psi}(\Theta)=-\int d^{4} x \int_{0}^{1} d s A(x ; s \Theta) \Theta(x)=\int d^{4} x L_{e f f}^{\psi}(U)-W_{W Z W}
$$

where the Wess-Zumino-Witten term $W_{W Z W}$ describes topological properties of $U$.

Eliminating external colour axial fields, $A_{\mu}=0$, we get the effective chiral Lagrangian $L_{\text {eff }}^{\psi}(U)$ arising from integration over fermions with $N_{F}$ flavors

$$
\begin{gathered}
L_{e f f}(U)=N_{F} t_{C}\left\{\frac{f_{0}^{2}}{4} D_{\mu} U D^{\mu} U^{-1}+\frac{1}{192 \pi^{2}}\left[\frac{1}{2}\left[U D_{\nu} U^{-1}, U D_{\mu} U^{-1}\right]^{2}-\left(U D_{\nu} U^{-1} U D^{v} U^{-1}\right)^{2}\right]\right. \\
+\frac{1}{96 \pi^{2}}\left(\left[U D^{\mu} U^{-1}, U D^{v} U^{-1}\right]\left(G_{v \mu}+U G_{\nu \mu} U^{-1}\right)+G_{\mu \nu} U G^{\mu v} U^{-1}\right)
\end{gathered}
$$

where the kinetic term contains a constant $f_{0}^{2}$ which is an analogue of the pion decay constant $f_{\pi}^{2}$.

\section{Soliton for $N_{F}=1$.}

Effective chiral lagrangian has the structure $L_{e f f}(U)=L_{k i n}(U)+L^{(4)}(U), L_{k i n}(U)$ - kinetic term with two derivatives, $L^{(4)}(U)$ - contains terms with 4 derivatives and do not depend on regularization scheme $R S$; the dimensional constant $f_{0}^{2}$ may look different in different $R S$, but in applications it should be taken from phenomenology. Derrick theorem is fulfilled, and $L_{e f f}$ can lead to static stable soliton solutions.

Next steps:

1. Choice of the ansatz for the color chiral field $U$

2. Choice of the background gluon field

Step 1. Natural boundary condition for color chiral field $U: U(x) \longrightarrow \mathbf{I}$, as $|x| \rightarrow \infty$. So $R^{3}$ is compactified to $S^{3}$ and we have mapping $U: S^{3} \rightarrow S U\left(N_{c}\right)$.

We are looking for topological solitons with nontrivial topological charge (winding number). In the case of $(3+1)$-dimensional models this can be done only for two symmetry groups [19] $G_{1}=\operatorname{diag}\left[S O(3)_{I} \otimes S O(3)_{S}\right], G_{2}=\operatorname{diag}\left[S O(2)_{I} \otimes S O(2)_{S}\right]$ 
The fields invariant against transformations from $G_{1}$ are spherically-symmetric fields of the hedgehog type, winding numbers $Q=1,2, \ldots$ Those invariant under group $G_{2}$ are axisymmetric configurations with winding numbers $Q \geq 2$. For the Skyrme model $E_{Q=2}\left(G_{2}\right) \leq E_{Q=2}\left(G_{1}\right)$.

For the case $N_{F}=1$ we are looking for the configuration with minimal energy, so we choose the group $G_{1}=\operatorname{diag}\left[S O(3)_{I} \otimes S O(3)_{S}\right]$.

We consider the colour gauge group $S U(2)$ with antihermitian generators $T_{a}=\frac{\tau_{a}}{2 i}$, where $\tau_{a}$ are the Pauli matrices. Let us write the chiral field in the usual hedgehog way

$$
U=\exp i\left(\frac{x_{a} \tau_{a}}{R}\right) F(R)=\cos F+i \mathbf{r} \sin F, r_{a} r_{a}=r^{2}=1, r_{a} \tau_{a}=\mathbf{r}, r_{a}=\frac{x_{a}}{R}
$$

Step 2. Colour configurations $U$ are always associated with background colour field $G_{\mu}$ because of necessity to maintain colour gauge invariance. In this respect, the case of colour solitons is quite different from the case of flavor solitons, where there is no flavour gauge invariance, and the external flavour gauge field can be eliminated from the chiral action. The background colour field should be chosen according to the colour configurations $U$ under consideration. The gluonic vacuum $\Psi_{0}$ is characterized by the condensate

$$
C_{g}=\left(\Psi_{0}, \frac{g^{2}}{4 \pi^{2}} O_{\mu \nu}^{a} O^{\mu v a} \Psi_{0}\right) \cong \frac{g^{2}}{4 \pi^{2}} G_{\mu \nu}^{a} G^{\mu v a} \neq 0
$$

that is by the non-zero vacuum expectation value of the Yang-Mills lagrangian for the full quantum field $O_{\mu}$ represented by the background vacuum field $G_{\mu}$ in our approximation. According to phenomenological descpription $C_{g} \succ 0, C_{g} \approx 0.04 G e V^{4}[20]$, so $G_{\mu}$ is a chromomagnetic field in the real case of $\mathrm{SU}(3)$ gauge group. The vacuum field strength $G_{k l}$ in the temporal gauge $G_{0}=0$ is constant up to a time independent gauge transformation.

We consider the simplest case of a chromomagnetic vacuum background field, when it is an Abelian-type field which is a product a coordinate vector field $V_{k}$ and a $\mathrm{SU}(2)$ color vector $n^{a}$

$$
G_{k}^{a}=V_{k} n^{a}, V_{k}=-\frac{1}{2} V_{k l} x_{l}=-\frac{1}{2} \varepsilon_{k l m} x_{l} v_{m} B, G_{k}=g G_{k}^{a} \frac{\tau_{a}}{2 i}
$$

where $n^{a}$ is a constant unit vector in the colour space, $v_{m}$ is a constant unit vector in coordinate space, $v_{m} B=\frac{1}{2} \varepsilon_{m l k} V_{l k}$ is the vacuum chromomagnetism and $B$ is related to the condensate $C_{g}=\frac{g^{2}}{2 \pi^{2}} B^{2}$. In the vacuum all directions $n^{a}$ and $v_{l}$ are equivalent, so that it is necessary to average over them at the end.

We are now able to right down the Effective colour static Lagrangian

$$
\begin{gathered}
L_{e f f}\left(U, G_{k}\right)=-N_{F} \frac{f_{0}^{2}}{4}\left[2\left(\left(\partial_{R} F\right)^{2}+2 \frac{\sin ^{2} F}{R^{2}}\right)+\frac{2}{9} g^{2} B^{2} R^{2} \sin ^{2} F\right]+\frac{N_{F}}{96 \pi^{2}}\left[\left(\left(\partial_{R} F\right)^{2}+2 \frac{\sin ^{2} F}{R^{2}}\right)^{2}+\right. \\
\left.\frac{16}{225} g^{4} B^{4} R^{4} \sin ^{4} F+\frac{2}{9} g^{2} B^{2} R^{2}\left(\left(\partial_{R} F\right)^{2}+3 \frac{\sin ^{2} F}{R^{2}}\right) \sin ^{2} F\right]-\frac{N_{F}}{72 \pi^{2}} g^{2} B^{2} \sin ^{2} F \\
-\frac{N_{F}}{24 \pi^{2}}\left[\frac{2 \sin ^{2} F}{R^{2}}\left(\partial_{R} F\right)^{2}+\frac{\sin ^{4} F}{R^{4}}+\frac{2}{9} g^{2} B^{2} \sin ^{4} F\right]
\end{gathered}
$$


The static energy or mass

$$
M=-4 \pi \int d R R^{2} L_{e f f}\left(U, G_{k}\right)
$$

must be finite. Near the origin $R=0$ soliton soliton function $F(R)$ behaves in the same manner as in the Skyrme model $F(R) \approx k \pi-b R$

The asymptotic behavior of $F(R)$ at large $\mathrm{R}$ is determined by the gluon condensate $C_{g}$

$$
F \rightarrow\left(f_{0} R\right)^{-\frac{3}{2}} \exp \left(-\frac{\pi}{3} \sqrt{\frac{C_{g}}{2}} R^{2}\right), R \rightarrow \infty
$$

\section{Group structure of $E \chi$-transformations}

In order to consider quark-antiquark and quark-quark composites on equal footings one should introduce eight-component spinors $\Psi$ constructed from ordinary Dirac spinors $\psi$ We consider the Dirac operator $D$ for massless quarks $q$ with background vector $V_{\mu}$ and axial vector $A_{\mu}$ fields and look for impact of transformations, which mixes particles and antiparticles and introduce fields $\omega$ with diquark quantum numbers

$$
\delta q=-\alpha q-\gamma_{5} \omega C \bar{q}^{T}, \delta \bar{q}^{T}=-\alpha^{*} \bar{q}^{T}-\gamma_{5} \omega^{*} C q
$$

on the quark lagrangian $L=\frac{1}{2}\left(\bar{q} D q-q^{T} D^{T} \bar{q}^{T}+q^{T} C \Phi q+\bar{q} \bar{\Phi} \bar{q}^{T}\right)$.

The extended chiral group $E \chi$ is the group of all gauge and chiral transformations leaving quark lagrangian invariant, if external fields are transformed accordingly. In absence of external fields, $E \chi$ is the group of global color and flavor transformations leaving free quark lagrangian invariant. The basic Dirac spinor $\Psi$ is 8-component: $\Psi=\left(\begin{array}{c}q \\ \bar{q}^{T}\end{array}\right)$

and the Dirac lagrangian $L$ can be rewritten as $L=\frac{1}{2} \Psi^{T} \widehat{F} \Psi$ with $\widehat{F}$ represented in the block form

$$
\widehat{F}=\left(\begin{array}{cc}
C \Phi & -\hat{D}^{T} \\
\hat{D} & \bar{\Phi} C
\end{array}\right)=-\widehat{F}^{T},
$$

where $\hat{D}=i \gamma^{\mu}\left(\partial_{\mu}+V_{\mu}+\gamma_{5} a_{\mu}\right)$ is the Dirac operator with external fields, "T" means transposition and $\Phi=\gamma^{\mu}\left(\phi_{5 \mu}+\gamma_{5} \phi_{\mu}\right)$ contains external vector diquark fields, $\bar{\Phi}=\gamma_{0} \Phi^{+} \gamma_{0}$.

To avoid difficulties with the Majorana spinors in finding a counterpart of $\widehat{F}$ in the Euclidean path integral over $\Psi$, one should take special care keeping in mind that what is necessary for chiral actions is only to calculate $\operatorname{det} \widehat{F}$. To this end we use the hermitian operator $\widehat{G}=\left(\begin{array}{cc}\hat{D} & \bar{\Phi} \\ \Phi & \hat{D}_{c}\end{array}\right), \hat{D}_{c}=C^{-1} \hat{D}^{T} C$, with $\operatorname{det} \widehat{G}=\operatorname{det} \widehat{F}$ and required positivity properties. Thus, having in mind the chiral anomaly and related effective action, one should study transformations of operator $\widehat{G}$ induced by quark transformation $\delta \Psi$

$$
\delta \Psi=-\Omega \Psi, \widehat{G} \rightarrow \widehat{G}^{\prime}=\exp \left(-\Xi+\gamma_{5} \Theta\right) \widehat{G} \exp \left(\Xi+\gamma_{5} \Theta\right)
$$


where antihermitian matrices $\Omega, \Xi, \Theta$ are given by

$$
\begin{gathered}
\Omega=\rho_{11}\left(\alpha+\gamma_{5} \chi\right)+\rho_{22}\left(\alpha^{*}-\gamma_{5} \chi^{*}\right)+ \\
\rho_{12}\left(\xi+\gamma_{5} \omega\right) C+\rho_{21}\left(-\xi^{*}+\gamma_{5} \omega^{*}\right) C \\
\Xi=\rho_{11} \alpha+\rho_{22} \alpha^{*}+\rho_{12} \xi+\rho_{21} \xi^{*}, \Theta=\rho_{11} \chi-\rho_{22} \chi^{*}+\rho_{12} \omega-\rho_{21} \omega^{*}
\end{gathered}
$$

We introduced notation: $\rho_{a b}$ is a $2 \times 2$ block matrix with elements $\left(\rho_{a b}\right)_{c d}=\delta_{a c} \delta_{b d}$.

We have $\alpha^{+}=-\alpha, \chi^{+}=-\chi, \xi^{T}=-\xi, \omega^{T}=\omega$. The quark baryon number is $\hat{b}=$ $\frac{1}{3}\left(\rho_{11}-\rho_{22}\right)=\frac{1}{3} \hat{\rho}_{3}$.

Transformations with $\Xi$ do not change the quark path integral, while transformations with $\Theta$ induce chiral anomalies. The Lie algebras with $\Xi+\gamma_{5} \Theta$ and $\Xi+\Theta$ are isomorphic. For $N_{c}$ colors and $N_{f}$ flavors, generators $\Xi+\Theta$ are in algebra of $U(2 N), N=N_{c} N_{f}$. Non-anomalous generators $\alpha$ are in algebra of $S U(N)$ and include color $S U\left(N_{c}\right)$; the leftright group $\mathbf{G}_{L R}=S U(N)_{L} \times S U(N)_{R}$ has $\alpha, \chi$ as generators, while $(\Xi+\Theta)_{s p}=\rho_{11} \alpha+$ $\rho_{22} \alpha^{*}+\rho_{12} \omega-\rho_{21} \omega^{*}$ is in algebra of symplectic group $\operatorname{Sp}(2 N)$, because $(\Xi+\Theta)_{s p}^{T} i \rho_{2}+$ $i \rho_{2}(\Xi+\Theta)_{s p}=0$.

In $E \chi$ non-anomalous generators $\Xi$ are in algebra of the orthogonal group $O(2 N)$ and include in addition to block diagonal generators $\alpha$ also non-diagonal generators $\xi$, which arise from non-commutativity of anomalous generators $\Theta_{L R}=\rho_{11} \chi-\rho_{22} \chi^{*}$ and $\Theta_{s p}=\rho_{12} \omega-\rho_{21} \omega^{*}$. Anomalous generators $\Theta_{s p}$ of the symplectic group $\operatorname{Sp}(2 N)$ belong to the coset $S p(2 N) / S U(N)$. We see that there are two distinguished subgroups $\mathbf{G}_{L R}$ and $S p(2 N)$ of $E \chi$ with the same block diagonal non-anomalous generators $\Xi^{0}=\rho_{11} \alpha+\rho_{22} \alpha^{*}$ and different anomalous parts $\Theta_{L R}$ and $\Theta_{s p}$, which do not require introduction of additional non-anomalous generators $\xi$. In the case of entire group $E \chi$, anomalous generators $\Theta$ include both $\chi$ and $\omega$ and belong to the coset $S U(2 N) / O(2 N)$.

\section{Effective chiral lagrangian for field $U$}

The chiral field is $U=\exp \Theta$. The calculation of effective lagrangian by integration of anomaly follows the standard bosonization procedure. After eliminating external color axial fields, $A_{\mu}=0$, we get the chiral action for $2 N$ internal degrees of freedom $W(U)=$ $W_{e f f}(U)-W_{w z}$ with an effective lagrangian $L_{e f f}(U)$ :

$$
\begin{gathered}
L_{e f f}(U)=t_{c, f}\left\{\frac{f_{\omega}^{2}}{4} D_{\mu} U D^{\mu} U^{-1}+\frac{1}{384 \pi^{2}}\left[\frac{1}{2}\left[U D_{v} U^{-1}, U D_{\mu} U^{-1}\right]^{2}-\left(U D_{v} U^{-1} U D^{v} U^{-1}\right)^{2}\right]\right. \\
\left.+\frac{1}{192 \pi^{2}}\left(\left[U D^{\mu} U^{-1}, U D^{v} U^{-1}\right]\left(\tilde{G}_{v \mu}+U \tilde{G}_{v \mu} U^{-1}\right)+\tilde{G}_{\mu v} U \tilde{G}^{\mu v} U^{-1}\right)\right\}, \\
D_{\mu}=\partial_{\mu}+\left[\tilde{G}_{\mu}, *\right], \quad \tilde{G}_{\mu}=\rho_{11} G_{\mu}+\rho_{22}\left(-G_{\mu}^{T}\right)+\rho_{12} \bar{\Phi}+\rho_{21} \Phi
\end{gathered}
$$

The kinetic term depends on a phenomenological parameter $f_{\omega}^{2}$.

The structure of $L_{e f f}(U)=L_{k i n}(U)+L^{(4)}(U)$ is the same as in the previous case. 


\section{Solitons in the symplectic group}

By next step we must find the ansatz for the color chiral field $U$ and then consider the choice of the background gluon field. We shall restrict ourselves to the case of scalar diquark as a natural partner in the formation of the nucleon. We are looking for the fields invariant against transformations from $G_{1}$, which are spherically-symmetric fields of the hedgehog type with winding number $Q=1$.

Let us consider the symplectic group $S p(2 N)$ with $\alpha$ in $S U(N), N=N_{c} N_{f}$. The chiral field $U_{s p}$ is a mapping from $S^{3}$ to $\Theta_{s p}=\rho_{+} \omega-\rho_{-} \omega^{*}$ with $\omega=\omega^{T}$. For the whole group (with both $\alpha$ and $\omega$ ) such mappings belong to $\pi_{3}=\mathbf{Z}$. Excluding $\alpha$ we get:

(a)Two or three colors, one flavor: $\omega=\lambda_{a} \omega_{a}, a=1,3,4,6,8$, no spherical solitons;

(b) Two colors, two flavors: $\omega=\Lambda_{b} \omega_{b}, \Lambda_{b}$ are nine traceless symmetrical matrices $\sigma_{2} \times \tau_{2}$ and $\sigma_{\tilde{k}} \times \tau_{\tilde{l}} ; \tilde{k}, \tilde{l}=0,1,3 ;$ no spherical solitons;

(c) Three colors, two flavors; non-anomalous part $\Xi_{s p}$ is given by $12 \times 12$ matrix with $\alpha$ in $S U$ (6) built on $S U$ (3) matrices $\lambda_{a}$ and flavor Pauli matrices $\tau_{k}$, while anomalous part $\Theta_{s p}$ together with $\Xi_{s p}$ span $\operatorname{Sp}(12)$ algebra. Symmetric matrix $\omega$ contains fields with diquark quantum numbers associated with both symmetrical matrices $\lambda_{a}^{S} \times \tau_{k}^{S}$ and both antisymmetrical ones $\lambda_{a}^{A} \times \tau_{2}$. Antisymmetric matrices

$$
\lambda^{A}=\left(\lambda_{2},-\lambda_{5}, \lambda_{7}\right) \equiv\left(O_{k}\right) ;\left(O_{k}\right)_{i j}=-i \varepsilon_{k i j}
$$

These are the generators of a $\overline{3}$-representation, which is equivalent to the spin one representation of an $S U(2)$ group

In color $O(3)$ algebra we combine with unit coordinate vector $r_{k}, r_{k} r_{k}=1$ into $\hat{r}=O_{k} r_{k}$. We retain only those parameters $\omega$, which enter with generators of $O(3)$, introduce the shape function $F_{s p}(R)$ and write the spherically-symmetric ansatz made out of fields $\vartheta_{k}, \vartheta_{k}^{*}$ with diquark quantum numbers for the color chiral field as

$$
\begin{gathered}
\Theta_{s p}=\rho_{+} i \tau_{2} O_{k} \vartheta_{k}-\rho_{-} i \tau_{2} O_{k} \vartheta_{k}^{*} \equiv i \tau_{2} \eta \hat{r} F_{s p}, \eta=\rho_{1} \cos \chi-\rho_{2} \sin \chi \\
U_{s p}=\exp \Theta_{s p}=1+i \tau_{2} \eta \hat{r} \sin F+\hat{r}^{2}(\cos F-1), R^{2}=x_{k} x_{k},
\end{gathered}
$$

assuming that $\chi$ is constant. Isospin matrices $I_{k}=\left(\rho_{3} \tau_{1}, \tau_{2}, \rho_{3} \tau_{3}\right)$ commute with $\Theta_{s p}$.

\section{Choice of background gluon field}

A single color soliton is defined as a soliton in the background of the vacuum chromomagnetic field $G_{\mu}^{a}=V_{\mu} N^{a}$, which depend on constant color unit SU(3) - vector $N^{a}, N^{a} N^{a}=1$. Soliton in a particular color domain is correlated with its background field. This correlation disappears, when we average over different color domains and important phenomenological parameter - gluon condensate forms. Consider $D_{\mu} U=\partial_{\mu}+\left[\hat{G}_{k}, U\right]$. Doubled gluon field

$$
\hat{G}_{k}=\rho_{11} G_{k}+\rho_{22}\left(-G_{k}^{T}\right), G_{k}=V_{k} \hat{N}, \hat{N}=\lambda_{a} N_{a}, N_{a} N_{a}=1
$$

$U=\exp \Theta$ and $\Theta$ contains only antisymmetric matrices $\lambda^{A}$. Thus, we should take $N=N^{A}$ antisymmetric, otherwise symmetric $N^{S}$ would take $\Theta$ out of $O(3)$ algebra. Note, that $\left(N^{A}\right)^{3}=N^{A}$. We see that $\operatorname{tr}\left(N^{A}\right)^{3}=0$. It follows that cubic gluon condensate is zero. 
In order to preserve the form of $U$ we have to restrict $\Xi_{s p}$ to a common subgroup $O(3)$ of $S U(3) \times S U(3)^{*}$. As we see, this is possible for vacuum background field $G_{k}^{a}=V_{k} \hat{N}, \hat{N}=$ $\lambda_{a} N_{a}, \operatorname{tr} \hat{N}^{3}=0$ in the gauge $G_{0}^{a}=0$.

Then $\hat{G}_{k}=\rho_{11} G_{k}+\rho_{22}\left(-G_{k}^{T}\right)=\left(\rho_{11}+\rho_{22}\right) V_{k} \tilde{N}$, where $\tilde{N}=O_{l} N_{l}, \tilde{N}^{3}=\tilde{N}$.

In the vacuum all directions $n^{a}$ and $v_{l}$ are equivalent, so that it is necessary to average over them at the end.

The effective color chiral static Lagrangian $L_{e f f}(U)$ for diquark solitons take the following form after averaging:

$$
\begin{gathered}
L_{e f f}(U)=-\frac{1}{4} N_{f} f_{\omega}^{2}\left\{2 F^{\prime 2}+2\left(\frac{2}{R^{2}}+\frac{1}{9} \pi^{2} C_{g} R^{2}\right) \sin ^{2} F+\left(\frac{4}{R^{2}}+\frac{1}{9} \pi^{2} C_{g} R^{2}\right) \sin ^{4} \frac{F}{2}\right\}- \\
\frac{1}{48 \pi^{2}}\left\{\left(F^{\prime 2}-\frac{\sin ^{2} \frac{F}{2}}{R^{2}}\right)+\frac{1}{18} \pi^{2} C_{g} R^{2}\left(F^{\prime 2}+\sin ^{2} F(\cos F-1)\right)\right\}+ \\
\frac{1}{96 \pi^{2}}\left(2 F^{\prime 4}-8 \frac{\sin ^{2} \frac{F}{2}}{R^{2}}\left\{F^{\prime 2}-3 \frac{\sin ^{2} \frac{F}{2}}{R^{2}}+\frac{1}{72} \pi^{2} C_{g} R^{2}\left(F^{\prime 2}+24 \sin ^{2} \frac{F}{2}\right)\right\}-\right. \\
\left.\frac{1}{225} \pi^{4} C_{g}^{2} R^{4}\left(\sin ^{2} F+4 \sin ^{2} \frac{F}{2}\right)\right)
\end{gathered}
$$

From finitness of mass functional $M(F)=-\int d^{3} x L_{e f f}$ we get the behavior of solitonic shape function at origin $\frac{F(R)}{2} \rightarrow \pi k, k$-integer.

Topological charge $t_{\chi}(U)$ for a soliton $U$ in the left-right subgroup of $E \chi$ is related to the quark baryon number $\hat{b}=\hat{\rho}_{3} / N_{c}$

$$
t_{\chi}(U)=\frac{1}{2} \frac{1}{24 \pi^{2} N_{c}} \int d^{3} x \varepsilon_{i j k} \operatorname{tr}\left\{\rho_{3} U D_{i} U^{+} U D_{j} U^{+} U D_{k} U^{+}\right\}
$$

and coincides with the baryon number of soliton $U$.

Finiteness of soliton mass follows from the static effective lagrangian $L_{\text {eff }}$ and asymptotic behavior of the shape function $F(R)$ at large $R$.

Asymptotics of $F(R)$ is defined by the kinetic term. The kinetic term averaged over directions $N^{k}$ and $v_{t}$ of background vacuum field $G_{l}^{k}=V_{l} N^{k}, V_{l}=-\frac{\pi}{2 i} \varepsilon_{l j t} r_{j} v_{t} R \sqrt{\frac{C_{g}}{2}}$ in $O(3)$ color and coordinate spaces takes the following form

$$
\left.K=\frac{1}{4} N_{F} f_{\omega}^{2}\left\{2 F^{\prime 2}+2\left(\frac{2}{R^{2}}+\frac{1}{9} \pi^{2} C_{g} R^{2}\right) \sin ^{2} F+\left(\frac{4}{R^{2}}+\frac{1}{9} \pi^{2} C_{g} R^{2}\right) \sin ^{4} \frac{F}{2}\right\}\right\}
$$

where $C_{g}$ is the gluon condensate. Thus, leading asymptotic behavior at large $R$ of the shape function $F(R)$ is governed by the similar equation as in the case of color $N_{F}=1$ quark soliton. We get the result

$$
F \rightarrow\left(f_{0} R\right)^{-\frac{3}{2}} \exp \left(-\frac{\pi}{3} \sqrt{\frac{C_{g}}{2}} R^{2}\right), R \rightarrow \infty
$$

which guarantees that the mass $M=-4 \pi \int d R R^{2} L_{\text {stat }}$ is finite for positive condensate $C_{g}$, i.e. for chromomagnetic vacuum field. 


\section{Conclusions}

We have shown that classically stable, finite mass topological solitons exist in the extended chiral group $E \chi$. In the case of three colors, two flavors their status is described by the chiral group $O(3)_{\tilde{L}} \times O(3)_{\tilde{R}}$. The vacuum background gluon field defines also an asymptotic behavior of the shape function $F(R)$; the field should be chromomagnetic. This pattern based on establishing mapping from $S^{3}$ to anomalous part $\Theta$ of $E \chi$ and using properties of vacuum background field can be followed in more complicated cases. This expansion of the world of color solitons is not accompanied by the widening of pure QCD gauge space: it is still $S U(3)$ and no additional gauge degrees $\xi$ were still required to accommodate solitons. However, these degrees $\xi$ are likely to appear, if vacuum is to be described by set of condensates. Diquark type solitons may be essential in discussion of baryon asymmetry, baryon number nonconservation and "electroweak baryons".

\section{References}

[1] Novozhilov Yu., Pronko A., Vassilevich D. Chiral and conformal anomalies of a quark path integral with diquark currents, Teoret. Mat. Fiz. 1994. Vol. 101. P.211-224.

[2] Novozhilov Yu., Pronko A.,Vassilevich D. Extended Chiral Transformations Including Diquark Fields As Parameters, Phys.Lett. (B). 1995. Vol. 343. P. 358-363.

[3] A.A.Andrianov, Yu.V.Novozhilov, Phys.Lett. B 153 (1985) 422

[4] A.Andrianov, Phys.Lett. 157B (1985) 425

[5] Andrianov A.A., Andrianov V.A., Novozhilov V.Yu., Novozhilov Yu.V. Spectral asymmetry, condensates and the QCD chiral lagrangian, Lett. Math. Phys. 1986. Vol. 11. P. 217-224.

[6] Adler S.L., Axial-Vector Vertex in Spinor Electrodynamics, Phys.Rev. 1969. Vol. 177. P. 2426-2438.

[7] Bell J.S., Jackiw R. A PCAC puzzle: $\pi^{0}->\gamma \gamma$ in the sigma model, Nuovo Cim. (A) 1969. Vol. 60. P. 47-61.

[8] Faddeev L., Niemi A. Stable knot-like structures in classical field theory, Nature 1997. Vol. 387. P. $58-61$.

[9] Battye R., Sutcliffe P., Knots as Stable Soliton Solutions in a Three-Dimensional Classical Field Theory, Phys.Rev.Lett. 1998. Vol. 81. P.4798-4801.

[10] Faddeev L., Niemi A., Magnetic Geometry and the Confinement of Electrically Conducting Plasmas, Phys.Rev.Lett. 2000. Vol. 85. P. 3416-3419.

[11] Y.M. Cho, Monopoles and Knots in Skyrme Theory, Phys.Rev.Lett. 87, 252001 (2001).

[12] Bae W.S., Cho Y.M., Kimm S.W., Extended QCD versus Skyrme-Faddeev theory, Phys.Rev.D. 2002. Vol. 65. P. 025005.

[13] Cho Y.M., Lee H.W., Pak D.G. Monopole Condensation in SU(2) QCD, Phys.Lett.B Vol. 2002. 525. 347-351. 
[14] Kaplan D.B. Constituent quarks as collective excitations of QCD, Phys.Lett.B 1990. Vol. 235. 163-169.

[15] Kaplan D.B. Qualitons, Nucl.Phys.B. 1991. Vol. 351. 137-160.

[16] Frishman Y., Hanany A., Karliner M. On the Stability of Quark Solitons in $Q C D,[$ hep-ph/9507206].

[17] Novozhilov V., Novozhilov Yu. Colour chiral solitons in low-energy QCD, Phys. Lett. (B). 2001. Vol. 522. P 49-56.

[18] Novozhilov V. Yu., Novozhilov Yu. V. Color Skyrmion in the Vacuum Field: Asymptotic Behavior, Stability, and the Possibility of Confinement, Teoret. Mat. Fiz. 2002. Vol. 131. P. 62-71.

[19] S.Coleman, Aspects of Symmetry, Cambridge Univ.Press, Cambridge U.K. 1985.

[20] Shifman M.A., Vainshtein A.I., Zakharov V.I. QCD and resonance physics. Theoretical foundations Nucl.Phys. B 1979 Vol. 147. P. 385-447. 